\title{
Gas flow in a microdevice with a mixing layer configuration
}

\author{
Sylvanus Yuk Kwan Lee ${ }^{1}$, Zeta Tak For Yu ${ }^{1}$, Man Wong ${ }^{2}$ and \\ Yitshak Zohar ${ }^{1}$ \\ ${ }^{1}$ Department of Mechanical Engineering, Hong Kong University of Science and Technology, \\ HKSAR, Clear Water Bay, Kowloon, Hong Kong, People's Republic of China \\ ${ }^{2}$ Department of Electrical and Electronic Engineering, Hong Kong University of Science and \\ Technology, HKSAR, Clear Water Bay, Kowloon, Hong Kong, People's Republic of China \\ E-mail: mezohar@ust.hk
}

Received 14 September 2001, in final form 9 November 2001

Published 11 December 2001

Online at stacks.iop.org/JMM/12/96

\begin{abstract}
We have designed an integrated microsystem with a mixing layer configuration, fabricated using standard micromachining techniques, in order to study fluid flows, in complex microfluidic systems, that are certain to find numerous important applications, especially in biomedical or chemical analysis. The device features two narrow and parallel channels merging smoothly into a wide channel downstream of a splitter plate, all $1 \mu \mathrm{m}$ in height, integrated with distributed pressure sensors. The characterization of the device included measurements of flow rate and pressure distribution for single-phase gas flow. Argon gas was passed either through one of the inlet channels, while the other was blocked, or through both inlet channels. Simple flow models of either a single straight microchannel or a pair of microchannels with different widths, connected in series, have been found to provide reasonable predictions of the evolving flow fields.
\end{abstract}

\section{Introduction}

Microfluidic systems have attracted much research interest since they not only provide tiny transducers for sensing and actuation but they also allow the study of flow phenomena dominated by surface effects [1]. Initially, the flow in straight microchannels received most of the attention because of its simplicity and the fundamental aspects involved. The results have indicated that gas flow in microchannels is indeed in the slip flow regime [2], different from the continuum regime of gas flow in macrochannels. This flow field, however, presents a rather simple balance between the pressure gradient and wall shear stress. Recently, the focus of research has shifted to more complex fluidic microsystems. Minor pressure losses in microchannels due to contraction/expansion sections [3], bends [4] or constriction elements [5] have been evaluated experimentally. Preliminary results have indicated that flow separation might occur in these microchannels, although Hele-Shaw flow is expected to develop due to the small channel height.

Mixing in fluids is another complex flow phenomenon, which is critical in a variety of systems, particularly in biomedical and chemical applications. In unbounded mixing layers, the velocity profile is unstable due to small perturbations, which grow and form large vortices. These structures control the mass and momentum transfer across the shear region. However, final mixing of the fluids is controlled by fine structures resulting from nonlinear interactions of the large vortices [6]. Clearly, in a microdevice, the presence of the walls close to the mixing region would have a major impact on the flow evolution. A theoretical study of the use of bend-induced vortices to stir the fluid and enhance the mixing process has shown that chaotic advection was observed under certain conditions [7]. Numerical modelling of chaotic advection thermally actuated by a bubble-pump has also demonstrated the possibility of fluid mixing [8]. The mixing characteristics of a T-type micromixer have been simulated, and the wall shear was found to play a significant role [9]. Diffusion in microcapillaries was experimentally investigated by separating the main flow into partial flows, which were laterally alternated in order to increase the surface between the liquids $[10,11]$. In another approach, the liquid flow was passed through a serpentine microchannel as a means 


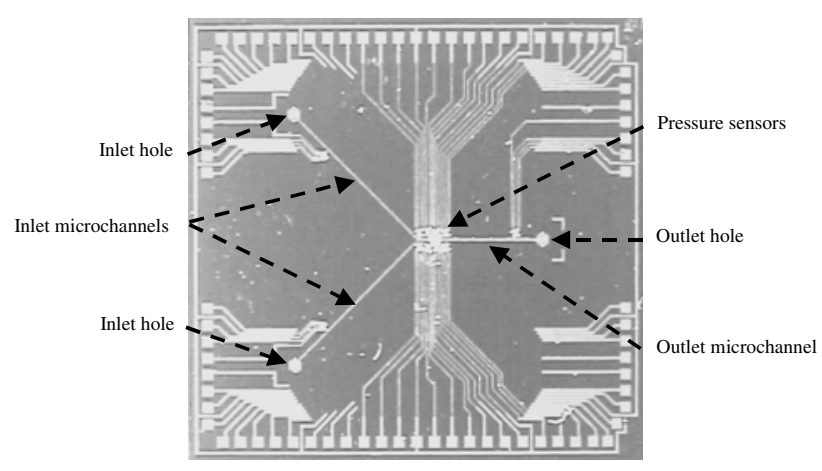

Figure 1. An overall photograph of a fabricated microdevice with a mixing layer configuration showing the merging microchannels, pressure sensors, two inlets and one outlet, and metal interconnects.

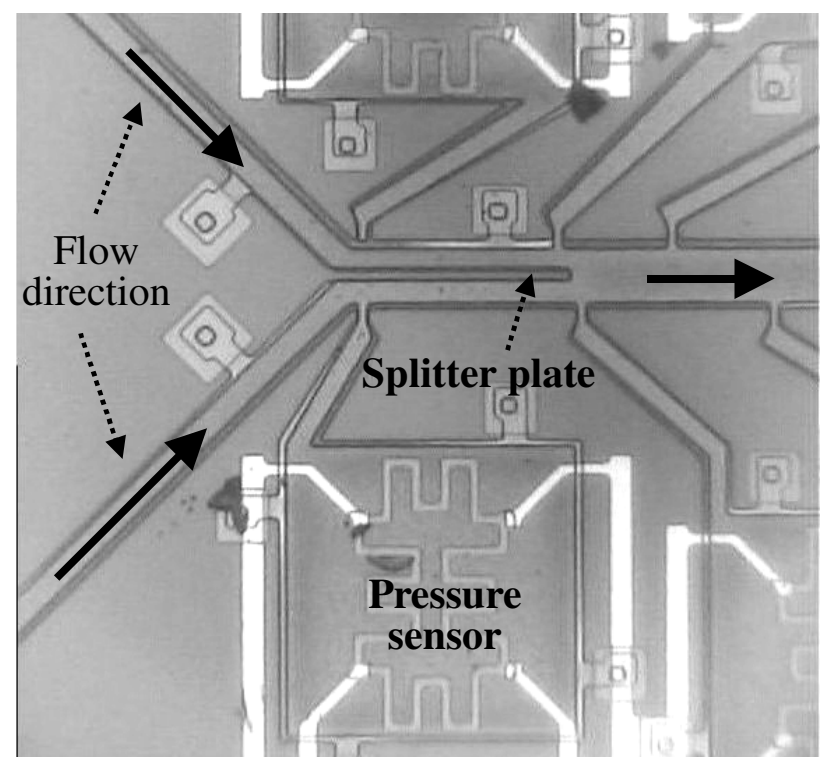

Figure 2. A close-up image of the mixing region showing the splitter plate and the merging channels with densely distributed pressure sensors.

of implementing chaotic advection to enhance fluid mixing [12]. Active perturbations, provided by a pressure source/sink system, were also used to enhance the mixing between two fluid streams [13]. The results have shown that chaotic-like distortions at the interface could enhance the effectiveness of the mixing between the streams.

In this paper, an integrated microsystem consisting of a pair of narrow microchannels merging into a wider channel has been fabricated in a mixing layer configuration to study the flow field in such microsystems.

\section{Device design and fabrication}

The microdevices have been designed in a mixing layer configuration [14]. Each device includes two identical inlet channels, $20 \times 1 \times 3050 \mu \mathrm{m}^{3}$ in nominal dimensions, which merge into a single outlet channel, with the dimensions of $40 \times 1 \times 2000 \mu \mathrm{m}^{3}$, downstream of a splitter plate as shown in figure 1. A close-up picture of the splitter plate, which has a width of about $6 \mu \mathrm{m}$ and a length of $80 \mu \mathrm{m}$, is shown in

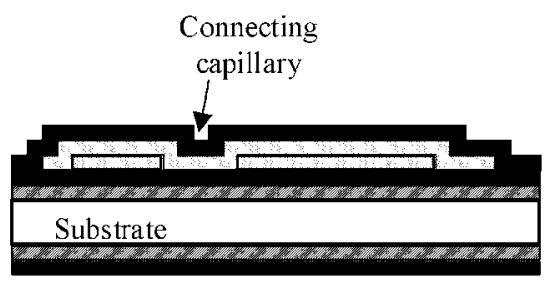

(a)

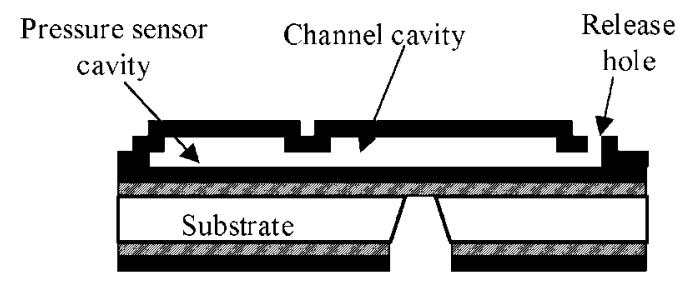

(b)
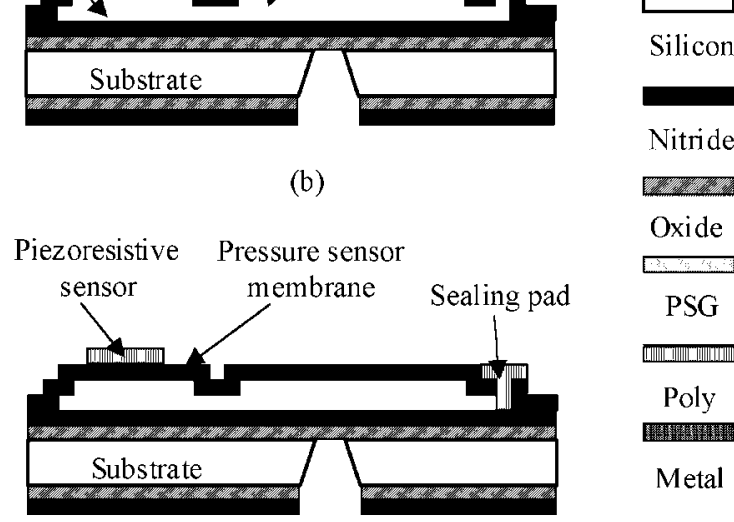

Nitride

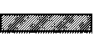

Oxide
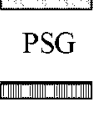

Poly

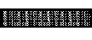

Metal

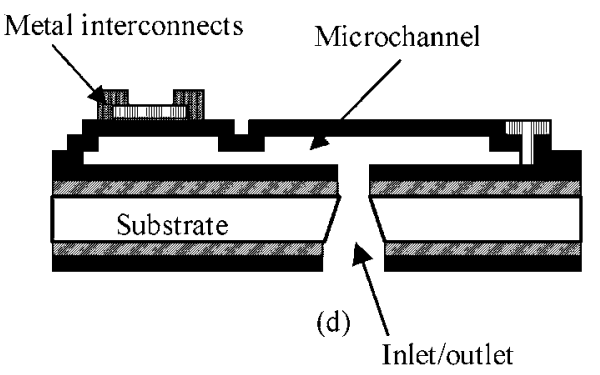

Figure 3. Schematic diagram of the cross sections of the major fabrication steps of the microdevices.

figure 2. The inlet channels include bends, at an angle of $45^{\circ}$ to provide the minimum separation distance of $4 \mathrm{~mm}$ between the two inlets required by the external system.

Pressure microsensors were distributed sparsely along the straight sections of the microdevices for reference, and densely around the mixing region for detailed measurements (see figure 2). The sensors are based on four piezoresistive sensing elements arranged in a Wheatstone bridge configuration. They are attached to square membranes, with an area of $100 \times$ $100 \mu \mathrm{m}^{2}$, and the chambers underneath are connected to the main channel via small capillaries. Any pressure difference across the membrane results in its deflection, thus straining the sensing elements and changing their resistance. The voltage change due to the resistance change is then recorded, and the pressure difference can be estimated using a calibration curve accordingly.

Figure 3 shows schematic cross sections of the main fabrication steps. The fabrication starts with the depositions 


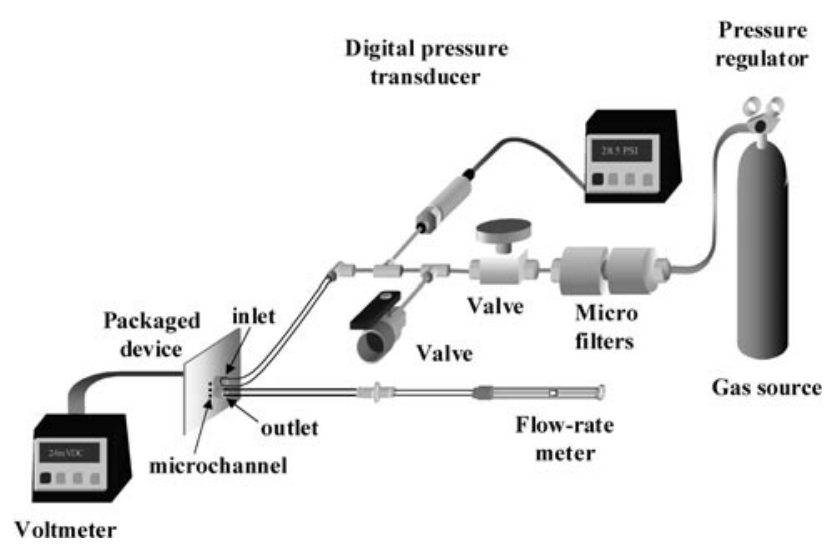

Figure 4. A schematic illustration of the experimental set-up.

of a $0.2 \mu \mathrm{m}$ thick thermal oxide and a $0.3 \mu \mathrm{m}$ thick low-stress nitride thin film on a silicon substrate for insulation. Two $0.5 \mu \mathrm{m}$ thick phosphosilicate glass (PSG) films were deposited, using low-pressure chemical vapour deposition (LPCVD), and patterned to form the channel and sensor chambers. The PSG films were selected as the sacrificial layers to provide a short etching time. The phosphorous content in the PSG determines the etch rate of the sacrificial layer; the higher the proportion of phosphorous, the faster the etch rate. In this paper, the phosphorous content is $6 \%$. The pattern of the second PSG layer is similar to that of the first, except for the distributed pads along the sides of the channel patterns which are used as etching holes. Next, the structural layer of a $1 \mu \mathrm{m}$, low-stress nitride film was deposited by LPCVD to form the channel walls and ceiling, as well as the sensor membranes (figure 3(a)). Anisotropic bulk etching was used to form the inlet/outlet holes from the back of the wafer, using the oxide/nitride double-layer stack as an etch mask. Following the opening of the release holes, the PSG sacrificial layer was etched in $49 \%$ hydrofluoric acid (HF) (figure 3(b)). This step is very critical since the release time cannot be too long, due to the fact that concentrated HF also etches silicon nitride at a rate of $0.04 \mu \mathrm{m} \mathrm{min}{ }^{-1}$. Therefore, the interval distance between the adjacent release holes should be designed properly. The time required to release all the cavities in the current device was less than $10 \mathrm{~min}$. Then, a polysilicon layer, $0.5 \mu \mathrm{m}$ thick, was deposited by LPCVD to serve as the sensing material and to seal the release holes. It was boron doped and patterned to form the piezoresistors on the sensor membrane (figure 3(c)). The LPCVD process resulted in a very low pressure in the chambers, and the diaphragms were deflected downwards under the ambient atmospheric pressure. Finally, for the interconnections a $0.8 \mu \mathrm{m}$ thick aluminium layer was sputtered, patterned and sintered to complete the whole fabrication process (figure $3(d)$ ).

\section{Experimental set-up}

Figure 4 shows a schematic diagram of the experimental set-up. Argon gas was supplied and controlled from a pressurized cylinder with a regulator, and then passed through the microdevice under an inlet pressure of up to 60 psi.

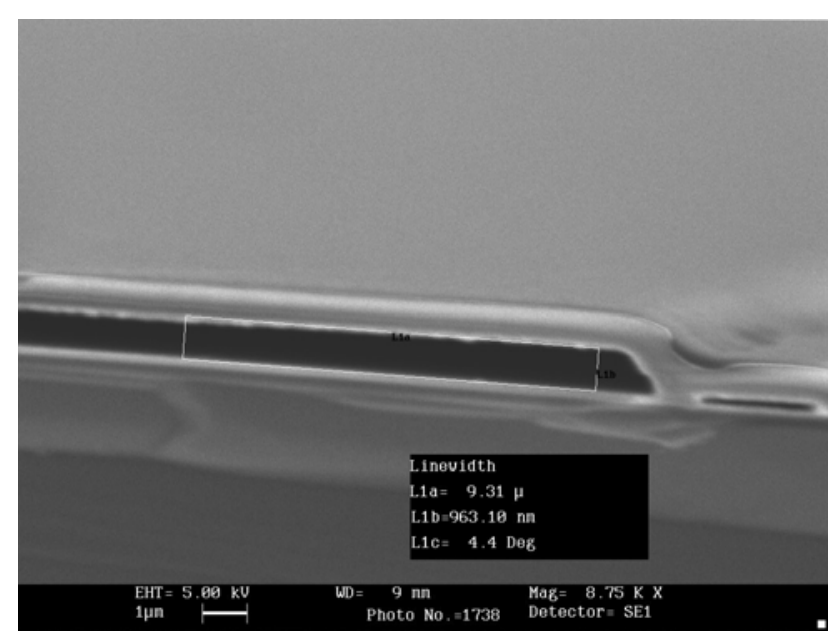

Figure 5. A SEM micrograph of a fabricated microchannel cross section.

The device outlet was connected to a glass syringe for flow rate measurement, and the integrated pressure sensors were connected to a voltmeter to measure the Wheatstone bridge output of each sensor due to the pressure difference across its membrane.

The volume flow rate was determined visually as a meniscus of water travelling past the marked scale on a syringe with volume of $10 \mu \mathrm{l}$ as a function of time. Multiplying this rate by the connecting gas density of $1 \mathrm{~atm}$ yields the mass flow rate. During the sacrificial etch for release, the nitride boundaries on the top and bottom were also slightly etched. Thus, the channel height is larger than the thickness of the sacrificial layer. Since the flow rate is a cubic function of the channel height, it is very important to measure the height accurately. Hence, a few microchannels were cut and scanning electron microscopy (SEM) micrographs of the cross sections were taken, as shown in figure 5 , to determine the height of the microdevices. The average height measured from the images was found to be about $0.98 \mu \mathrm{m}$. Since the tested microchannels were fabricated simultaneously with the microchannels used for taking the SEM images, the heights of all the microdevices are assumed to be the same.

The experiments were first conducted by driving the gas through each branch separately, while blocking the inlet of the other branch, measuring both flow rate and pressure distribution. The experiments were repeated with both inlet branches connected together to the gas source. Prior to any measurements, all the sensors were calibrated.

\section{Mass flow rate measurements and calculations}

Argon gas flow rate measurements were conducted to investigate several features of the micromixer device: (1) the effect of the bend; (2) the effect of the splitter plate; (3) the effect of the merger of two streams when both inlets are connected together to the gas source; (4) the effect of sudden expansion when one inlet is connected to the gas source while the other is blocked. In order to provide a reference case for comparison, the mass flow rate is theoretically calculated for 


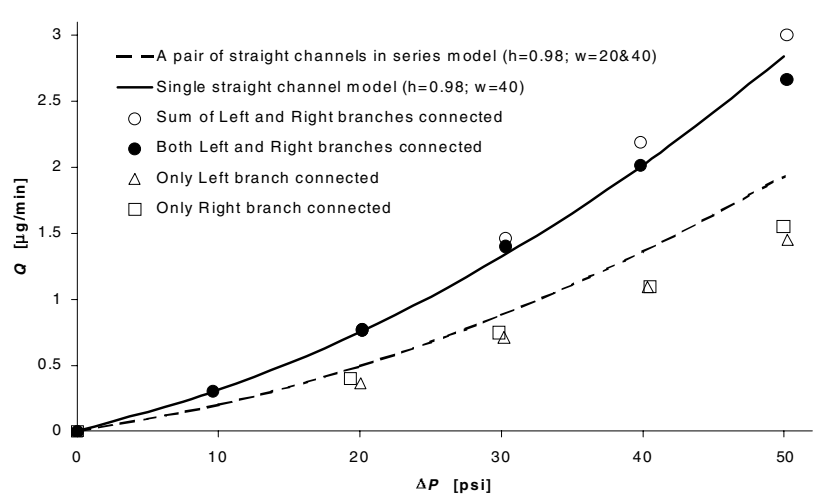

Figure 6. A comparison between measured and calculated mass flow rates for either a single branch or both inlet branches connected to the gas supply.

a straight and uniform microchannel that has the same overall length as the micromixer channel.

\subsection{Calculations of mass flow rate through a microchannel}

It is not easy to calculate the flow field when one inlet branch is blocked. However, when both narrow inlet branches are connected to the gas source, two streams of fluids merge into the wide outlet microchannel. Since the total width of the two inlet branches is identical to the width of the outlet microchannels, the microdevice with a mixing layer configuration can be considered as a uniform and straight microchannel if the bends and the friction on the inner sidewalls of the channels are negligible. The minor loss due to a double-turn bend of $90^{\circ}$ in a microchannel was found to be too small to be detected [4]. The present microdevice includes a single turn of $45^{\circ}$, and the minimum aspect ratio is 20. Therefore, a two-dimensional (2D) model of a straight microchannel should provide good reference data to evaluate the various features of the current microdevice. Accounting for the compressible and slip flow effects, the mass flow rate $Q_{m}$ in a straight and uniform microchannel under a driving pressure drop is given by [15]

$$
Q_{m}=\frac{H^{3} W P_{o}^{2}}{24 R T L \mu}\left[\left(\frac{P_{i}}{P_{o}}\right)^{2}-1+12 K n_{o}\left(\frac{P_{i}}{P_{o}}-1\right)\right]
$$

where $H, W$ and $L$ are the channel height, width of outlet channel and total length from inlet to outlet, $P_{i}$ and $P_{o}$ are the inlet and outlet pressure, and $T, \mu$ and $R$ are the fluid temperature, viscosity and specific gas constant, respectively. The outlet Knudsen number $K n_{o}=\lambda_{o} / H$, based on the outlet mean free path $\lambda_{o}$, is about $K n_{o}=0.06$ for argon at an exit pressure of $1 \mathrm{~atm}$. The mass flow rate calculations based on equation (1) for a microchannel, with dimensions of $40 \times 0.98 \times 5050$ $\mu \mathrm{m}^{3}$, are plotted by the full curve in figure 6 .

\subsection{Measurements of the mass flow rate}

Pressure-driven, mass flow rate measurements were carried out for three different configurations: (a) right inlet blocked and left inlet connected; (b) left inlet blocked and right inlet

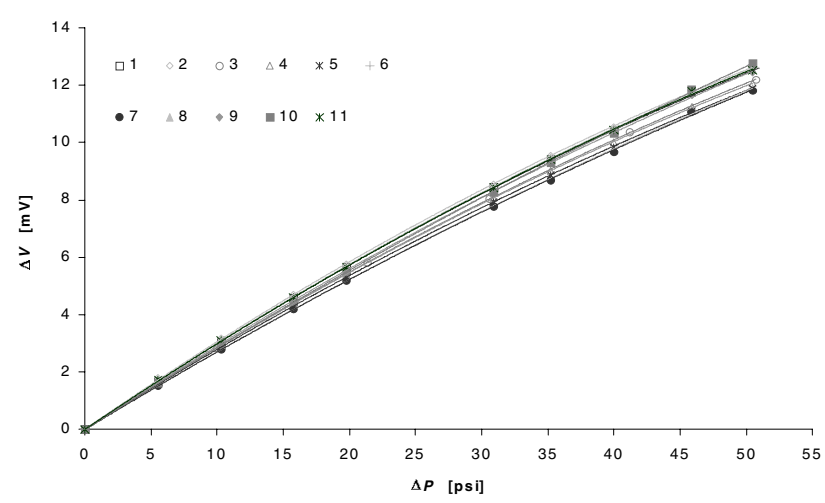

Figure 7. Calibration curves of the integrated pressure microsensors.

connected; (c) both left and right inlets connected to the gas source. The results are compared with the analytical predictions in figure 6 . The theoretical curve, calculated for a channel with a width of $40 \mu \mathrm{m}$, agrees most closely with the measured mass flow rate when both inlet branches are connected to gas source. This confirms that it is not only the loss due to the bends that is negligible, but also the loss to the extra sidewalls. Moreover, the two streams seem to merge smoothly, with no losses, such that the straight and uniform channel model is adequate.

The measured mass flow rate through one branch is almost identical to the other, indicating that both inlet branches are hydrodynamically symmetric as geometrically designed. In a 2D model, the mass flow rate depends linearly on the channel width as in equation (1). Therefore, the sum of the mass flow rate through two channels, $20 \mu \mathrm{m}$ wide, is equal to the flow rate through a single channel $40 \mu \mathrm{m}$ in width. The sum of the mass flow rate through each inlet branch, while the other is blocked, is slightly higher than the measured flow rate when both branches are connected together. Since both inlet branches are identical, this indicates that the resistance to the flow in a channel with a sudden expansion, from $20 \mu \mathrm{m}$ to $40 \mu \mathrm{m}$, is lower than the resistance to the flow in a uniform and narrow channel, $20 \mu \mathrm{m}$ wide. It is important to note, however, that the flow through a single inlet branch is not equivalent to a flow through a standard sudden expansion at the splitter plate trailing edge. Although the other inlet branch is blocked, secondary flow develops in the branch due to the pressure difference between the splitter plate trailing edge and the blocked-branch inlet. This secondary flow would result in a small minor loss. Consequently, the measured flow rate through a single branch $20 \mu \mathrm{m}$ wide, with an expansion to $40 \mu \mathrm{m}$, is only marginally higher than the calculated flow rate in a straight channel with a uniform width of $20 \mu \mathrm{m}$, as is evident in figure 6.

\section{Streamwise pressure distributions}

All the pressure sensors were calibrated prior to the pressure distribution measurements, and the calibration curves are depicted in figure 7 . The sensor sensitivity was on average about $0.13 \mathrm{mV} / \mathrm{psi} / \mathrm{V}$, sufficient for the pressure range tested 


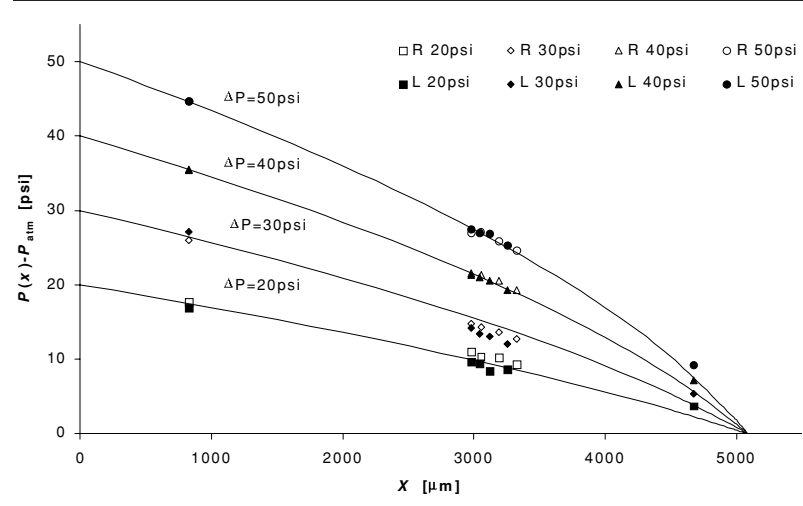

(a)

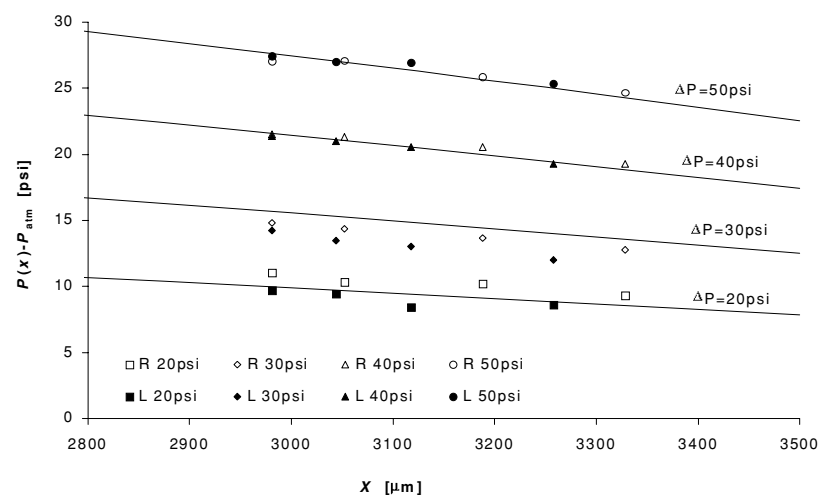

(b)

Figure 8. Calculated and measured pressure distributions, $(a)$ along the microdevice and $(b)$ around the mixing region, when both branches are connected to the gas source.

in this paper. The accuracy of pressure measurements is estimated to be within $\pm 3 \%$ of the nominal value. In parallel to the flow rate measurements, pressure distributions were measured either for both branches or for a single branch (the other being blocked) connected to the gas source. Since the flow rates confirmed that the two inlet branches are symmetric, it is possible to combine data points from either side of the channel.

\subsection{Pressure distributions with both inlet branches connected}

The agreement between the calculated and measured mass flow rates suggests that the flow in this configuration is similar to the flow in a straight microchannel. Pressure distributions should provide a more critical test of this similarity. The same analytical model which has been used for the flow rate calculations, can also be utilized for calculating the streamwise pressure distribution $P(x)$ along a straight microchannel [15],

$$
\begin{aligned}
& \frac{P(x)}{P_{o}}=-6 K n_{o}+\left\{\left(6 K n_{o}+\frac{P_{i}}{P_{o}}\right)^{2}-\left[\left(\frac{P_{i}^{2}}{P_{o}^{2}}-1\right)\right.\right. \\
& \left.\left.+12 K n_{o}\left(\frac{P_{i}}{P_{o}}-1\right)\right]\left(\frac{x}{L}\right)\right\}^{1 / 2}
\end{aligned}
$$

where $x$ is the streamwise distance along from the channel inlet. Obviously, the pressure is independent of the channel width since the flow is two dimensional. The calculated nonlinear streamwise pressure distributions (full curves) are compared in figure 8 with pressure measurements (symbols) when both inlets are connected to a gas source under a driving pressure of up to 50 psi. When the gas is forced through both inlet branches, the pressure decreases monotonically along each branch from the inlet to the splitter plate trailing edge due to friction (figure $8(a)$ ), and similarly from the splitter plate to the channel outlet. A close-up comparison of the pressure field around the splitter plate (figure $8(b)$ ) reveals an excellent agreement between the measured and calculated distributions for a straight channel. This indicates that the pressure on either side of the trailing edge is equal such that both streams merge together smoothly to fill up the outlet channel. Hence, the pressure continues to decrease monotonically through the transition from two branches to a single outlet channel. This further confirms that the flow development along the micromixer, when both inlets are connected together, can be predicted very well using the straight microchannel model.

\subsection{Pressure distributions with one inlet branch connected (the other blocked)}

When the gas is forced through only a single branch, the flow field is expected to be significantly more complicated. The flow around the splitter plate trailing edge cannot be considered as a simple sudden expansion, since a secondary flow would most likely develop in the blocked branch as a result of the pressure gradient between the splitter plate and the branch inlet. Clearly, the mass flow rate upstream (narrow channel) and downstream (wide channel) of the splitter plate is the same. Hence, one straight microchannel model is not applicable in this case, and instead a model of two straight channels connected in series with a transition region could be more adequate.

In the simplest model of two straight channels, a transition pressure $P_{t}$ is assumed to be the outlet pressure of the upstream, $20 \mu \mathrm{m}$ wide, channel and the inlet pressure to the downstream, $40 \mu \mathrm{m}$ wide, channel. Hence, equation (1) can be used to calculate the mass flow rate in each channel. Equating the mass flow rate in the two channels, conservation of mass, yields the following equation for the transition pressure, $P_{t}$ :

$$
\begin{aligned}
& \frac{H^{3} W_{i} P_{t}^{2}}{24 \mu R T L_{i}}\left[\left(\frac{P_{i}}{P_{t}}\right)^{2}-1+12 K n_{t}\left(\frac{P_{i}}{P_{t}}-1\right)\right] \\
& =\frac{H^{3} W_{o} P_{o}^{2}}{24 \mu R T L_{o}}\left[\left(\frac{P_{t}}{P_{o}}\right)^{2}-1+12 K n_{o}\left(\frac{P_{t}}{P_{o}}-1\right)\right]
\end{aligned}
$$

where $W_{i}, L_{i}, W_{o}$ and $L_{o}$ are the upstream channel width and length and the downstream channel width and length, respectively. The transition Knudsen number does not constitute an extra unknown since it depends only on the pressure, $K n_{t}=P_{o} K n_{o} / P_{t}$ [15]. The transition pressure can then be calculated for any combination of inlet/outlet pressures and, subsequently, not only the flow rate but also the 


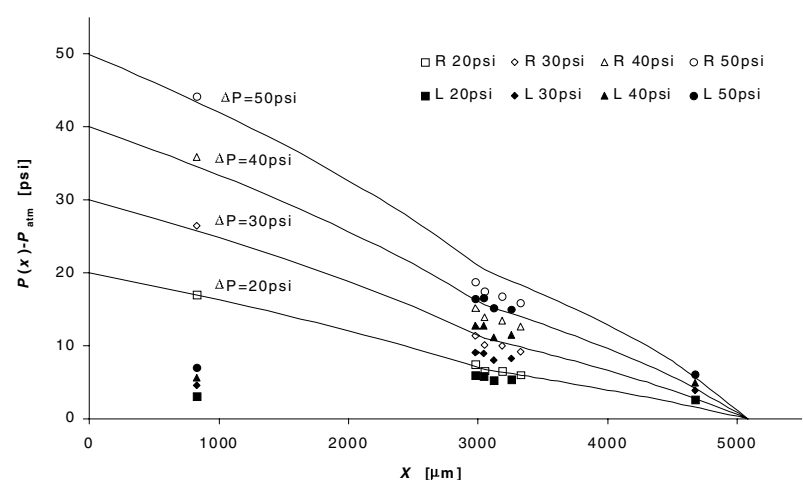

(a)

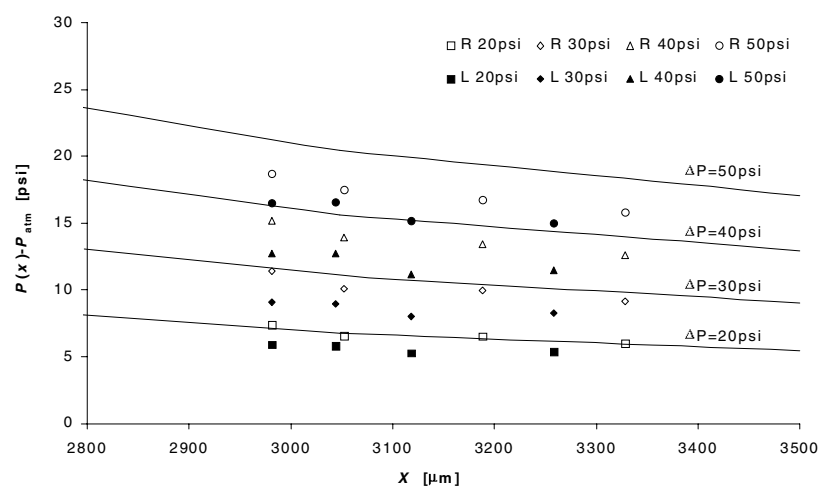

(b)

Figure 9. Calculated and measured pressure distributions, $(a)$ along the microdevice and $(b)$ around the mixing region, when only a single branch is connected to the gas source while the other is blocked.

pressure distribution in each channel can be calculated using equation (2):

$$
\begin{aligned}
& \frac{P(x)}{P_{t}}=-6 K n_{t}+\left\{\left(6 K n_{t}+\frac{P_{i}}{P_{t}}\right)^{2}-\left[\left(\frac{P_{i}^{2}}{P_{t}^{2}}-1\right)\right.\right. \\
& \left.\left.+12 K n_{t}\left(\frac{P_{i}}{P_{t}}-1\right)\right]\left(\frac{x_{i}}{L_{i}}\right)\right\}^{1 / 2} \\
& \frac{P(x)}{P_{o}}=-6 K n_{o}+\left\{\left(6 K n_{o}+\frac{P_{i}}{P_{o}}\right)^{2}-\left[\left(\frac{P_{i}^{2}}{P_{o}^{2}}-1\right)\right.\right. \\
& \left.\left.+12 K n_{o}\left(\frac{P_{i}}{P_{o}}-1\right)\right]\left(\frac{x_{o}}{L_{o}}\right)\right\}^{1 / 2}
\end{aligned}
$$

where $x_{i}$ is the streamwise distance from the channel inlet to the splitter plate trailing edge, and $x_{o}$ is the streamwise distance from the trailing edge to the channel outlet. In figure 9, the calculated distributions are compared with the experimental measurements. The agreement between the analytical and experimental results in figure $9(a)$ looks reasonable, and an even closer observation of the transition region in figure $9(b)$ reveals that the calculations over-predict the measured values by no more than $20 \%$. Furthermore, mass flow rate predictions consistent with this model are also just about $25 \%$ higher than the measured mass flow rate (figure 6). It is clear that a simple model based on straight channel, either single or double, cannot describe the complicated flow evolution when one branch is blocked. The pressure in the blocked branch is higher than the ambient pressure and lower than the pressure at the splitter plate trailing edge, suggesting the development of a secondary flow in the branch. Hence, a more sophisticated model is required to account for this secondary flow, which is probably the cause of a minor pressure loss resulting in a lower flow rate.

\section{Conclusions}

A microdevice with a mixing layer configuration, integrated with pressure microsensors, has been successfully fabricated and characterized. The device features two narrow channels merging smoothly into a wide channel downstream of a splitter plate. When both inlet channels are connected together to a gas source, the evolving flow field can be predicted accurately using a single straight and uniform microchannel model. When one channel is connected to the gas source, while the other is blocked, the model of a pair of microchannels in series over-predicts both the mass flow rate and pressure distribution by about $20-25 \%$. A more sophisticated model is required to account for the pressure loss at the splitter plate, resulting in lower pressure and flow rate.

\section{Acknowledgment}

This work has been supported by the Hong Kong Research Grant Council through RGC grant HKUST6012/98E.

\section{References}

[1] Ho C M and Tai Y C 1998 Micro-electro-mechanical systems (MEMS) and fluid flows Ann. Rev. Fluid Mech. 30 579-612

[2] Liu J Q, Tai Y C, Pong K C and Ho C M 1993 Micro-machined channel/pressure sensor systems for micro flow studies Proc. of the 7th Int. Conf. on Solid-State Sensors and Actuators, Transducers' 93 (Japan) pp 995-999

[3] Lee W Y, Lee S Y K, Wong M and Zohar Y 2000 Microchannels in series with gradual contraction/expansion Proc. Int. Mechanical Engineering Congress \& Exposition, Nov 2000 MEMS-Vol 2 pp 467-472

[4] Lee S Y K, Wong M and Zohar Y 2001 Pressure losses in microchannels with bends The 14th IEEE Int. Conf. on Micro Electro Mechanical Systems, Jan 2001 pp 491-494

[5] Lee W Y, Wong M and Zohar Y 2001 Flow separation in constriction microchannels Proc. 14th Int. Conf. on Micro Electro Mechanical Systems, MEMS'01 pp 495-498

[6] Ho C M and Huerre P 1984 Perturbed free shear layers Ann. Rev. Fluid Mech. 16 365-424

[7] Yi M and Bau H H 2000 The kinematics of bend-induced stirring in micro-conduits Proc. of Int. Mechanical Engineering Congress \& Exposition, Nov 2000 MEMS-Vol 2 pp 489-496 
[8] Evans J, Liepmann D and Pisano A P 1997 Planar laminar mixer Proc. IEEE. The 10th Ann. Int. Workshop on Micro Electro Mechanical Systems pp 96-101

[9] Gobby D, Angeli P and Gavriilidis A 2001 Mixing characteristics of T-type microfluidic mixers J. Micromech. Microeng. 11 126-132

[10] Koch M, Chatelain D, Evans A G R and Brunnschweiler A 1998 Two simple micromixers based on silicon J. Micromech. Microeng. 8 123-126

[11] Krog J P, Branebjerg J, Nielsen C R and Gravesen P 1996 Experiments and simulations on a micro-mixer fabricated using a planar silicon/glass technology Micro-ElectroMechanical Systems (MEMS) International Mechanical Engineering Congress and Exposition, ASME DSC-Vol 59 pp 177-82
[12] Liu R H, Stremler M A, Sharp K V, Olsen M G, Santiago J G, Adrian R J, Aref H and Beebe D J 2000 Passive mixing in a three-dimensional serpentine microchannel J. Micromech. Microeng. 9 190-197

[13] Lee Y, Tabeling P, Shih C and Ho C M 2000 Characterization of a MEMS-fabrication mixing device Proc. Int. Mechanical Engineering Congress \& Exposition, Nov 2000 MEMS-Vol 2 pp 505-511

[14] Lee S Y K, Lee K H N, Wong M and Zohar Y 2000 Design, fabrication and calibration of a mixing layer micro-device Int. Symposium on Smart Structures \& Microsystems, Hong Kong

[15] Arkilic E B, Schmidt M A and Breuer K S 1997 Gaseous slip flow in long microchannels J. Microelectromech. Sys. 6 $167-178$ 